\title{
MULTIFOCAL ELECTRORETINOGRAM, CENTRAL MACULAR THICKNESS AND VISUAL ACUITY IN DIABETIC MACULAR EDEMA FOLLOWING INTRAVITREAL INJECTION OF ANTI-VEGF
}

\author{
Syntia Nusanti ${ }^{1}$, Kirana Sampurna ${ }^{1}$, Ari Djatikusumo ${ }^{1}$, Anggun Rama Yudantha ${ }^{1}$, Joedo Prihartono ${ }^{1}$. \\ ${ }^{1}$ Universitas Indonesia, Cipto Mangunkusumo National Central General Hospital \\ ${ }^{2}$ Ophthalmology Department, Faculty of Medicine, Universitas Indonesia, Jakarta \\ ${ }^{3}$ Community Health Department, Faculty of Medicine, Universitas Indonesia, Jakarta, Indonesia
}

\begin{abstract}
Introduction: Diabetic Retinopathy $(D R)$ is one of the major cause of visual acuity deterioration in diabetic patients. The loss of central visual acuity in diabetic patients are mainly due to macula edema, which is found in 29\% diabetic patients with the over 20 years duration of disease. The purpose of this study is to assess the correlation between MfERG (amplitude and implicit time of $p$ wave), with central macular thickness (CMT) and visual acuity in diabetic macular edema (DME) patients following intra vitreal anti VEGF injection.
\end{abstract}

Methods: Single arm clinical trial. Thirty-three eyes of 33 DME patients (16 non-proliferative diabetic retinopathy and 17 non-high-risk proliferative diabetic retinopathy), received intravitreal bevacizumab 1,25mg. All patients underwent complete ophthalmic examination including ETDRS $V A$ testing, Sixty-one scaled hexagon MfERG and SD-OCT scan at baseline, 1-week and 1-month post-injection. Components of the first order kernel (N1, N2 and P1) in central $2^{\circ}$ were measured.

Result: MfERG showed reduction of $P 1$ amplitude $(P<0.05)$ at 1-week after injection followed by increase of $P 1$ amplitude $(P>0.05)$ at 1-month after treatment as compared to the baseline in all subjects. There was $19 \%$ improvement CMT and 0.2Logmar VA improvement in 1-month postinjection compared to the baseline $(P<005)$. This study showed no serious ocular adverse effects. There was no significant correlation among changes in visual acuity and changes in CMT or other MfERG parameters.

Conclusion: Intravitreal injection bevacizumab resulted in improvement of $V A$, reduction in CMT and mild improvement in the MfERG responses. Although VA changes did not correlate with reduced CMT nor with improved responses of MfERG, the combined use of SD-OCT and MfERG may be used to evaluate macular function in DME patient with worsened visual acuity post anti-VEGF injection.

\footnotetext{
Keywords: Diabetic Macular Edema; Multifocal Electroretinography; Optical Coherence Tomography; Visual Acuity

Cite This Article: NUSANTI, Syntia et al. Multifocal Electroretinogram, Central Macula Thickness and Visual Acuity in Diabetic Macular Edema Following Intravitreal Injection of anti-VEGF. International Journal of Retina, [S.I.], v. 4, n. 2, p. 150, sep. 2021. ISSN 2614-8536. Available at: <https://www.ijretina.com/index.php/iiretina/article/view/156>.doi: https://doi.org/10.35479/iiretina.2021.v ol004.iss002.156.
}

Correspondence to:

Syntia Nusanti,

Universitas Indonesia, Cipto

Mangunkusumo National Central

General Hospital;

syntia_nusanti@hotmail.com

\section{INTRODUCTION}

Diabetic Retinopathy (DR) is one of the major cause of visual acuity deterioration. Macula edema is the main cause of central visual loss in DR patients, which is found in $29 \%$ in diabetic patients. ${ }^{1}$. Descriptive study from Syabaniah et $\mathrm{al}^{5}$, showed that the incidence of DR from November 2010 October 2011 was $24.5 \%$ of all diabetic patients, 
which consisted of 565 patients with DR, 200 patients $(8.7 \%)$ with mild non-proliferative diabetic retinopathy and 207 patients $(11.37 \%)$ with moderate proliferative diabetic retinopathy.

Bevacizumab (Avastin ${ }^{\circledR}$, Greentech, Inc, San Francisco,CA) is categorized as an off-label drugs for DME treatment, however there were many reports that shown the effectivity and safety of bevacizumab for DME treatment. ${ }^{5}$ Michaelides et al., ${ }^{6}$ reported that in a prospective clinical trial study, the comparison between bevazicumab (BVZ) injection group with macular-laser photocoagulation group for 2 years which includes in BOLT Study, had shown that BVZ group had a better effectivity and improvement in visual acuity and macular thickness. A meta-analysis study by Reigner et al. $\stackrel{7}{ }$ also stated that intravitreal anti-VEGF injection was statistically superior compared to macular photocoagulation for visual acuity improvement.

The decrease in thickness of central macula should be followed with improvement of visual acuity, however several studies showed inconsistence results. Therefore scientists are eager to find another factors outside the anatomical structure of macular, that influence the improvement of visual acuity, such as photo transduction ability, photoreceptor cells function, in which all of them can be measured with electroretinogram (ERG). Sutter et al, developed a technique of ERG to focus in several area of retina, which was MfERG a noninvasive but sensitive method to simultaneously record several area in retina specifically in posterior pole. ${ }^{8}$

M-Sequence is a special algorithm to locate and show the damage area in the retina. Previous study also reported the importance of MfERG as a predictor in diabetic retinopathy. .10 The amplitude and implicit time of $P$ wave in MfERG have a hhigh sensitivity and specificity in diabetic retinopathy. $\cdot$ Study from Greenstein et al. ${ }^{11}$ and Farahsvasah et al. $^{12}$ reported that in patients with diabetic retinopathy, there were a significant decrease of $p$ wave amplitude and shorter implicit time in macular edema group, compared with non macular edema. The purpose of this study is to assess the correlation between MfERG (amplitude and implicit time of $p$ wave), with central macular thickness and visual acuity in DME patients following intra vitreal anti VEGF injection.

\section{METHOD}

This was single arm clinical trial, conducted in Eye Clinic, RS Cipto Mangunkusomo (RSCM) Kirana, in Jakarta, from November 2015 - March 2016. The sample of this study were subjects with type 1 and type $2 \mathrm{DM}, \geq 18$ years old with diagnosis of DME in all stages non proliferative diabetic retinopathy (NPDR), mild, moderate and severe and non-high risk proliferative diabetic retinopathy (PDR) according to Early Treatment of Diabetic Retinopathy Study (ETDRS), best corrected visual acuity between 1 and 35 according to ETDRS chart (equivalet to $1 / 60 \geq x \geq 6 / 12$ in Snellen Chart), the thickness of central macula measured by SD-OCT is more than $250 \mu \mathrm{m}$, and agreed to take part in the research and signed the informed consent.

The exclusion criteria in this study without, patients who had oxyhemoglobin level $(\mathrm{HbA} 1 \mathrm{c})>10.0 \mathrm{mg} / \mathrm{dl}$ for the last 6 months, history of ocular trauma, intraocular surgery for the last 6 months, laser retinal treatment for the last 6 months, abnormality in vitreretinal surface, had an opacity in refractive media which made the examination impossible and unable to visit for evaluation and follow up.

The samples were consecutively obtained until a total number of 35 samples were achieved. Each subject had a comprehensive examination from anamnesis and complete ophthalmology examination, followed by the next examination to check the central macular thickness using SD-OCT (Cirrus OCT, Carl Zeiss Meditec). This examination was performed in a dilated pupil with tropicamide $1 \%$ eye drop. Afterwards, full field electroretinography (FFERG) and MfERG were conducted using Metrovision ERG System. Before ERG examination, the pupil dilated using tropicamide $1 \%$ and corrected according to BCVA. ERG was performed according to ISCEV standard and guidelines by using jet electrode to acquired the signal.

First, the dark room adaptation was carried out for 20 minutes, then continued with scotopic 0.01, 3.0 and 3.0 Oscillatory Potential (OP). Light room adaptation was performed 
in 10 minutes before MfERG started. The stimulus given was consisted of 61 hexagonal spread over a 26 degree horizontal and 20 degree vertical in visual field. It was displayed on a 20 inch black and white screen with frame rate of $120 \mathrm{~Hz}$ and $1024 \times 768$ pixel resolution, $40 \mathrm{~cm}$ away from patients eyes. The amplitude and implicit time were measured in five retina area. Patients were asked to stay focus on the fixation mark (central area), meanwhile patients with decreased visual acuity were asked to focus on monitor. The measured parameter of MfERG were first order kernel.

The next procedure was bevazicumab intravitreal injection at procedure room. The procedure began by placing a topical anesthetic eye drop tetracaine (pantocain) into the injected eye. The $1.25 \mathrm{mg}$ bevazicumab was injected to supero-temporal region 3.55 $\mathrm{mm}$ (pseudophakia) or $4 \mathrm{~mm}$ (phakia) from limbus using $1 \mathrm{cc}$ syringe and $30 \mathrm{G}$ needle.

After 1 week and 1 month post injection, all patients returned to get a report examination include in best corrected visual acuity, microscopic slit-lamp, funduscopic, central macular thickness with SD-OCT and MfERG. This study had passed the ethical clearance review from ethical committee, Faculty of Medicine, Universitas Indonesia.

\section{RESULT}

Thirty-five subjects (35 eyes) were obtained consecutively in this study. Two were dropped out of the study. One subjects did not come for follow up at week four after injection and another one required additional therapy of laser grid macular and Pan-Retinal Photocoagulation (PRP) at 3 weeks after injections. Basic characteristics in our study included age, gender, duration for $\mathrm{DM}, \mathrm{HbA} 1 \mathrm{c}$ level, systolic and diastolic blood pressure and the degree of retinopathy are shown in Table 1.
Table 1. Basic characteristics

\begin{tabular}{lcc}
\hline \multicolumn{1}{c}{ Variable } & $\begin{array}{c}\text { Total } \\
(\mathrm{n}=33)\end{array}$ & $\begin{array}{c}\text { Percentage } \\
(\%)\end{array}$ \\
\hline Age (year) & $51.67 \pm 7.29$ & \\
Gender & & \\
Men & 16 & 48.5 \\
Women & 17 & 51.5 \\
Duration of DM (year) & $9(0.5 ; 21)$ & \\
Total cholesterol & $216.64 \pm$ & \\
(mg/dL) & 35.1 & \\
Systolic (mmHg) & $149.33 \pm$ & \\
& 17.1 & \\
Diastolic (mmHg) & $87(52 ; 100)$ & \\
HbA1c & $8.2(6.9 ; 10)$ & \\
Degree of retinopathy & & \\
Mild NPDR & 2 & 6.1 \\
Moderate NPDR & 8 & 24.2 \\
Severe NPDR & 6 & 18.2 \\
Non High-Risk PDR & 17 & 51.5 \\
Type of macular & & \\
edema & & 90.9 \\
Diffuse & 30 & 9.1 \\
Cystic & 3 & \\
\hline$\quad$ & & \\
\hline & &
\end{tabular}

Table 2. Clinical characteristics of patient (state unit for each variables)

\begin{tabular}{ll}
\hline Variable & Mean \\
\hline Uncorrected visual acuity (LogMar) & $0.72 \pm 1.59$ \\
Best corrected visual acuity (LogMar) & $0.51 \pm 1.86$ \\
CMT (um) & $372.9 \pm 1.3$ \\
Flicker wave Hz & $77.84 \pm 23.65$ \\
Scotopic 3.0 A-wave Hz & $-147 \pm 71.2$ \\
Scotopic 3.0 B-wave Hz & $266.2 \pm 140.1$ \\
Sum OP-wave Hz & $78.37 \pm 67.9$ \\
\hline
\end{tabular}

Table 1 showed that 17 out of 33 patients (51.5\%) had diabetic macular edema (DME) on non high-risk PDR. Table 2 shows that from 17 PDR patients, there were two extreme values of best corrected visual acuity (BCVA); best BCVA (1.78) and worst BCVA (0.2). Central macular thickness (CMT) data was distributed from 252 um minimum to 670 um maximum. FFERG examination showed reduction in all parameters (scotopic 3.0 A and B-wave, flicker wave and $O P$ wave). Minimum value of $O P$ wave was obtained from PDR patient, meanwhile maximum value of OP wave was shown in mild NPDR patient. The same result was found in extreme value of scotopic $A$ and B-wave amplitude. Of the total 33 patients, we did not find any signs of electronegative ERG 
b-wave/scotopic a-wave ratio of $<1$. Therefore, in this study, none of the patients had extensive retinal ischemia for more than $50 \%$. The normal b/a wave ratio in ERG test is $>1$, so this sentence actually is to describe that all the 33 subjects reveal their b/a wave ratio are $>1$, in a clinically PDR cases where it's linked to more severe degree of retina ischemia compared to Non-proliferative diabetic retinopathy (NPDR).
In this study there was no statistically significant difference in pre-injection visual acuity with the type of macular edema (cystic and diffuse type) $(p=0.997)$. The same result was found in type of diabetic retinopathy, there was no difference in visual acuity, CMT and all MfERG parameters (amplitude and implicit time P1, N1 and N2) pre-injection between the NPDR group compared with the PDR group.

Table 3. Comparation of pre-injection, 1 week post-injection and 1 month post-injection

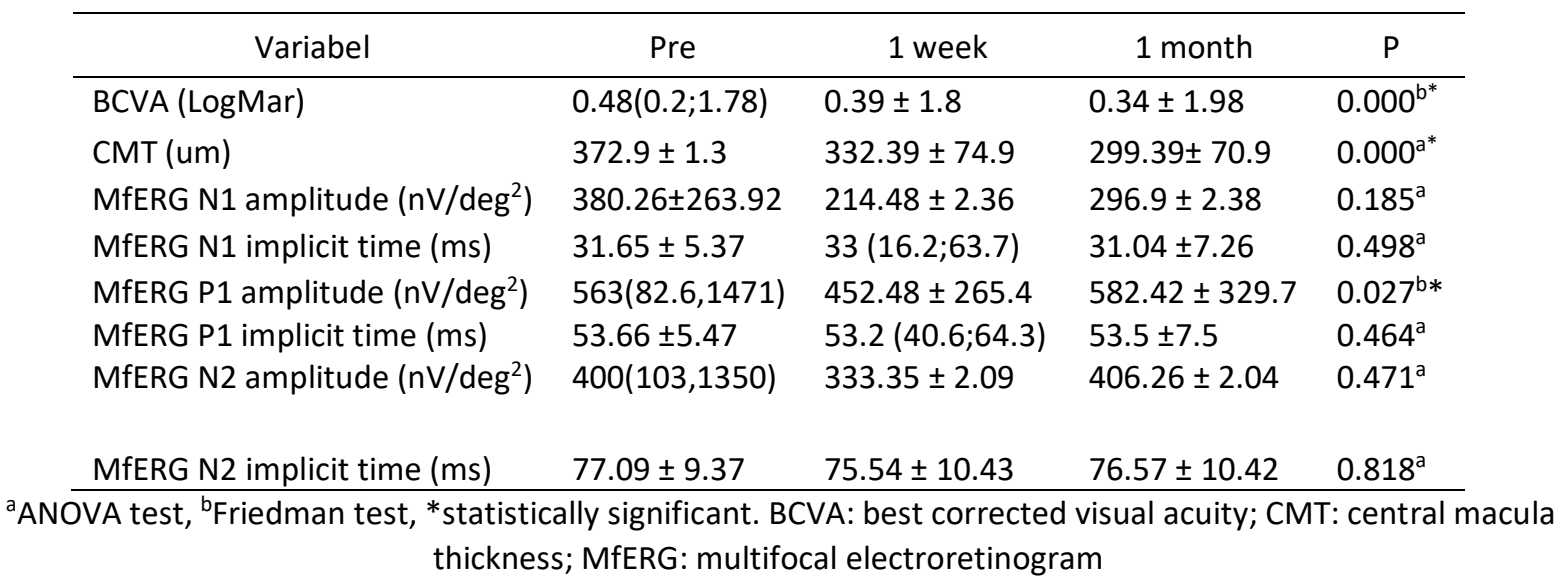

Table 3 showed the mean values of each parameter at baseline, one week and one month after intravitreal bevacizumab injection. The data distribution of BCVA was non normal distribution, using the Friedman test, there was a significant improvement in visual acuity between pre-injection, one week and one month post-injection $(p<0.05)$. Furthermore, from non-parametric Wilcoxon rank test, we found statistically significant improvement in visual acuity from pre-injection (baseline) compared to 1 week post-injection.

ANOVA test was used for CMT data. There was a significant reduction in CMT at one month post-injection $(p<0.05)$. Subsequently, a post-hoc analysis was performed using Bonferroni correction with a statistically significant reduction in CMT at baseline-one week $(p=0.042)$ and baseline-one month $(p=$
0.000 ) but it was not significant at one weekone month $(p>0.05)$. Meanwhile, the results of Friedman test on the P1 MfERG amplitude showed a significant change in amplitude at pre-injection, one week and one month after injection. Furthermore, using the Wilcoxon rank test was carried out. There was a significant decrease at one week after injection ( $p=0.008)$ followed by a significant improvement in the P1 amplitude at one month post-injection $(p=0.014)$.

Other MfERG parameters such as N1 wave amplitude, N2 amplitude, N1 implicit time and $\mathrm{N} 2$ implicit time showed statistically significant $(p>0.05)$ both in ANOVA test (normal distribution) and Friedman test (non normal distribution), therefore we did not do post-hoc analysis. 

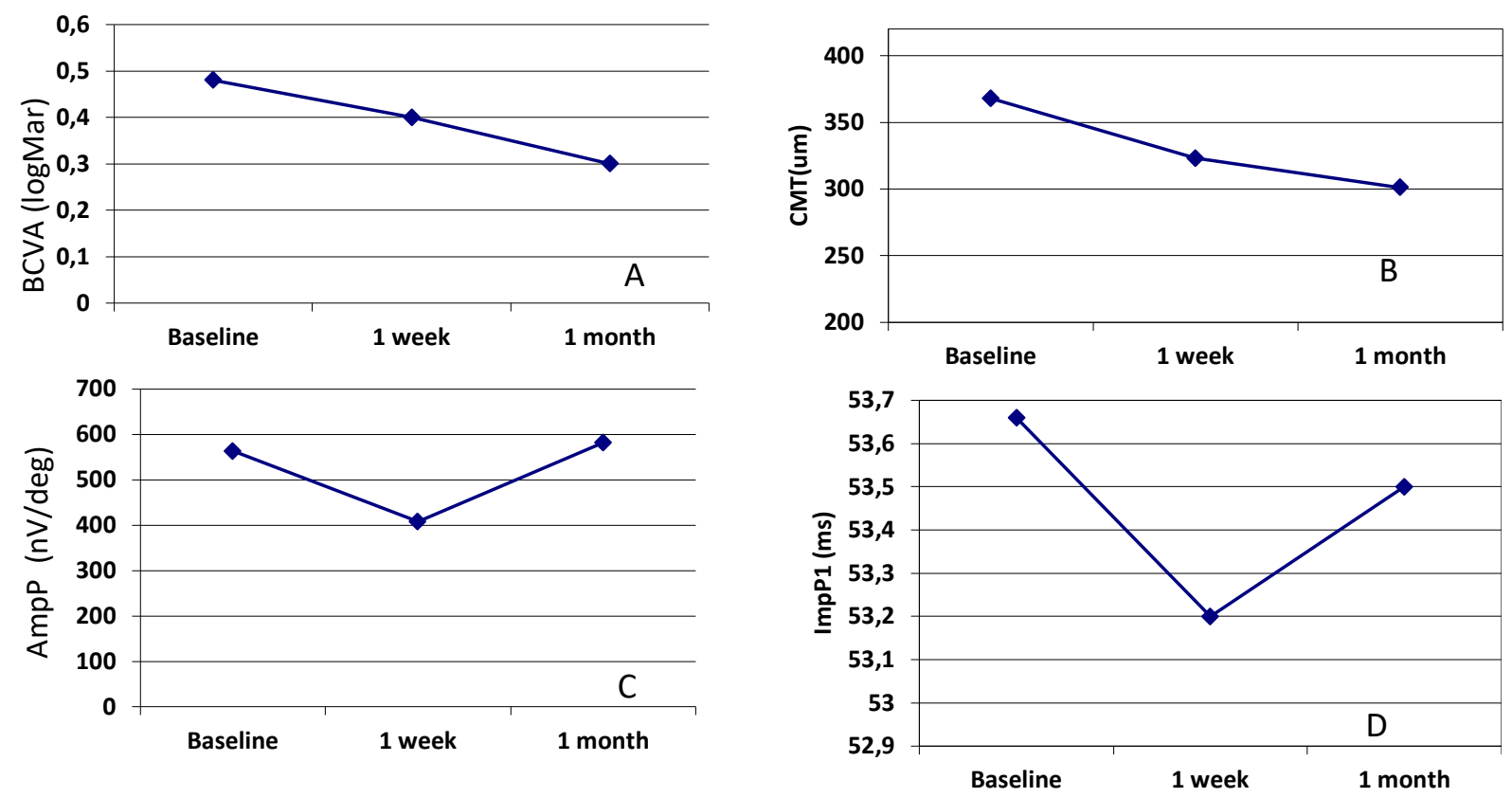

Figure 1. Graphics of mean based on examination time. A. Mean median value of BCVA based on examination time, B. Mean median value of CMT based on examination time. C. Mean value of P1 amplitude based on examination time; D. Mean value of implicit time P1 based on examination time.

The four graphs above showed improvement in mean visual acuity after bevacizumab injection accompanied by reduction in central macular thickness (CMT), improvement in P1 amplitude and P1 implicit time shortening. Mean visual acuity on improvement during one month follow-up was 2 lines ETDRS chart with a reduction in CMT by $19 \%$. From a total of 33 patients, 3 patients had decreased visual acuity and 5 patients had no change in visual acuity. In those three patients with decreased visual acuity, there were 2 patients with reduced CMT who experienced worsening of the MfERG parameter (P1 amplitude), whereas in one patient there was an improvement in MfERG parameters but CMT was better (410um).

In this study, an additional analysis was done to find correlation between the type of diabetic retinopathy and visual acuity, CMT, also MfERG parameters in each examination time. There was a significant difference $(p=0.307)$ between the implicit time of preinjection $\mathrm{N} 1$ and the type of diabetic retinopathy, but it was not significant at one week and one month post-injection. There was no significant difference for other parameters $(p>0.05)$. 


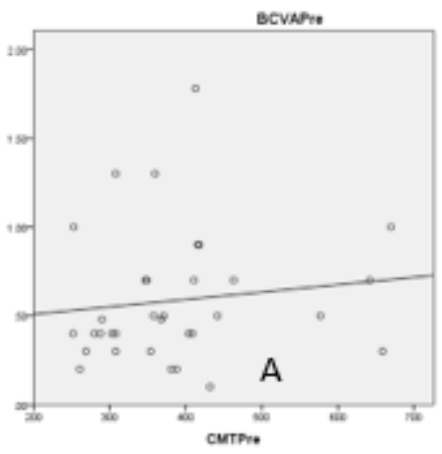

BCvapis
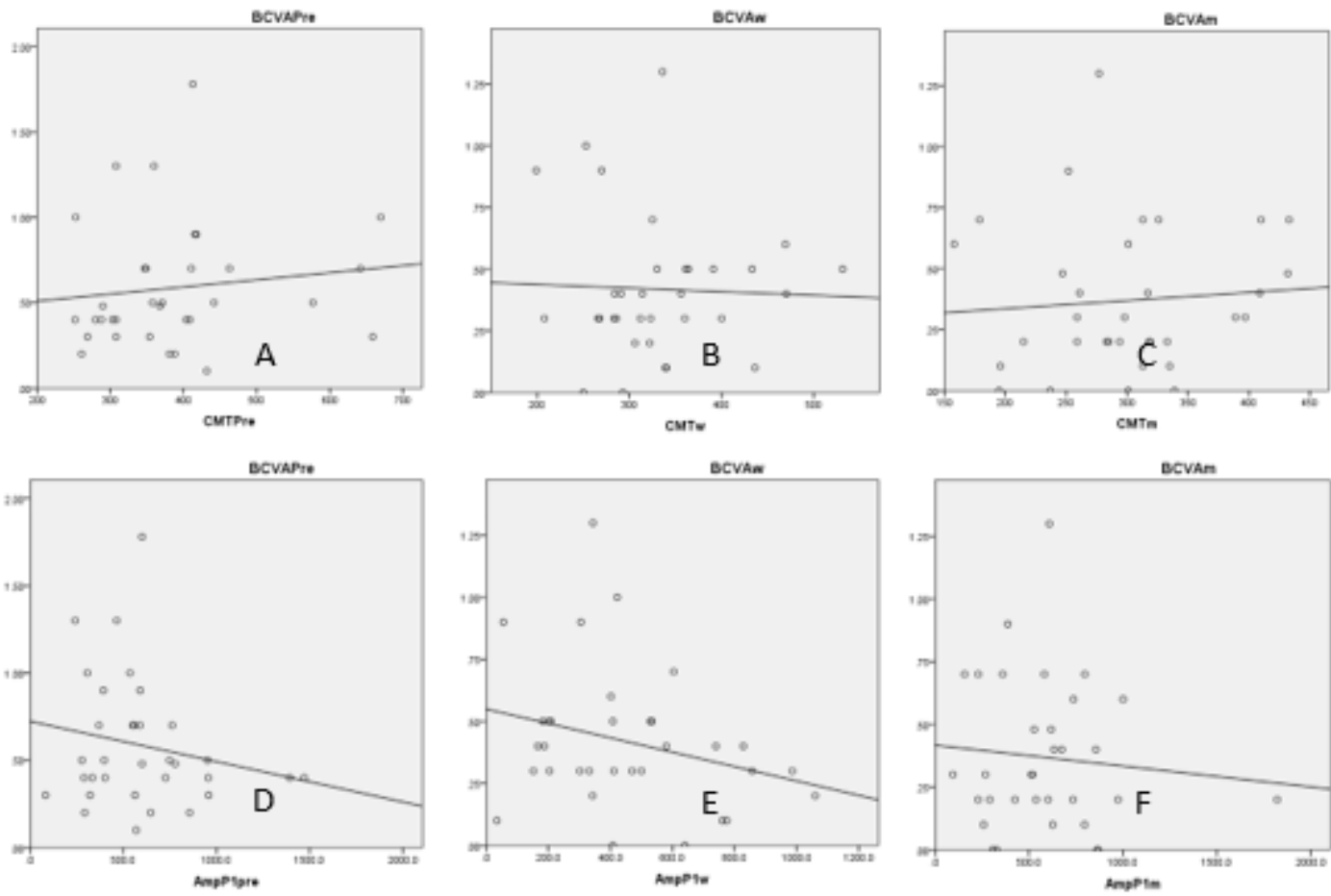

Figure 2. A, B, C. Scatter plot graphic. Correlation of pre-injection central macular thickness (CMT) and best corrected visual acuity (BCVA) (A), 1 week after bevacizumab injection (B), 1 month after intravitreal bevacizumab injection (C). D,E.F. Scatter plot graphic. Correlation of P1 amplitude and baseline BCVA (D), 1 week post-injection BCVA (E), 1 month post-injection BCVA (F).

Figures $2 a, 2 b$ and $2 c$ showed $a$ relationship between visual acuity improvement and CMT reduction but neither of them had a correlation at baseline, one week or one month after intravitreal bevacizumab injection. Meanwhile, figures $2 d, 2 e$ and $2 f$ indicated relationship between visual acuity improvement with an increase in P1 amplitude at baseline, 1 week and one month post-injection. Statistically, neither of them had a significant correlation $(R<0.03, p>0.05)$.

In addition, we analyzed the relationship among the diabetic macular edema (DME) type with visual acuity, CMT, and MfERG parameters at each examination time. Using the Mann-Whitney test, we found a significant difference between DME type and P1 implicit time at pre-injection $(p=0.307)$, but it was not significant at one week and one month post-injection. There was also significant difference between DME type with CMT and N1 amplitude at one month post-injection. There was no significant difference for other parameters $(p>0.05)$.

This study further analyzed the correlation between diabetic retinopathy type and DME type with changes in each parameter (visual acuity improvement, CMT reduction, amplitude improvement and implicit time shortening). Using the Mann-Whitney test, there was no significant correlation between the type of diabetic retinopathy and each parameter. Similar results were obtained for DME type and each parameter.

Further analysis was done to find correlation between changes in visual acuity (dependent variable) with changes in CMT and changes in MfERG parameters, respectively, at one week and 
one month post-injection. Correlation test between changes in CMT and changes in MfERG parameters was done thereafter. Tables 4 and 5 displayed no significant correlation between changes in visual acuity with changes in CMT or other MfERG parameters.

Table 4. Correlation of parameter changes between baseline with 1 week post-injection

\begin{tabular}{lccc}
\hline \multicolumn{1}{c}{ Eye parameters } & \multicolumn{3}{c}{ Eye parameters } \\
\cline { 2 - 4 } & CMT & P1 amplitude & P1 implicit time \\
\hline BCVA & & & 0.056 \\
Spearman's coefficient & 0.002 & 0.225 & 0.758 \\
P value & 0.991 & 0.207 & \\
CMT & & 0.080 & 0.224 \\
Spearman's coefficient & & 0.658 & 0.209 \\
P value & & 0.056 \\
P1 amplitude & & 0.755 \\
Spearman's coefficient & & & \\
P value & & \\
\hline
\end{tabular}

Table 5. Correlation of parameter changes between baseline with 1 month post-injection

\begin{tabular}{llll}
\hline \multicolumn{1}{c}{ Eye parameters } & \multicolumn{3}{c}{ Eye parameters } \\
\cline { 2 - 4 } & \multicolumn{1}{c}{ CMT } & P1 amplitude & N1 implicit time \\
\hline Visual acuity & & & 0,107 \\
$\quad$ Spearman's coefficient & 0,113 & 0.073 & 0,553 \\
$\quad$ P value & 0,531 & 0,687 & \\
Macular thickness & & 0,065 \\
$\quad$ Spearman's coefficient & 0,051 & 0,721 \\
$\quad$ P value & 0,208 & 0,170 \\
Amplitude score & & 0,344 \\
$\quad$ Spearman's coefficient & & \\
$\quad$ P value & & &
\end{tabular}

Data of complication were obtained from all study subjects including drop-out (35 subjects). Three patients (8.57\%) had mild complications (subconjunctival hemorrhage). Two other patients complained of floaters at one week post injection follow-up. The two subjects then underwent observation and did another follow-up examination at 4 weeks postinjection. There were no subjects who had increased eye pressure, severe inflammation of the front chamber or tractional retinal detachment.

\section{DISCUSSION}

Previous study reported findings of worsening of FFERG parameters in patients with diabetic retinopathy, especially in scotopic 3.0, OP and flicker $30 \mathrm{~Hz}$. In scotopic 3.0 the amplitude of $a$ and $b$ wave were measured. Both waves represent to interpret inner and outer retina layer function respectively. Flicker $3.0 \mathrm{~Hz}$ amplitude is the result of photoreceptor cone cells activities scattered throughout the retina, meanwhile OP wave amplitude resulted from amacrine, bipolar and muller cells activities. All subjects in this study had undergone FFERG examination. The result of this study showed the decrease in every FERG parameter (OP amplitude, $a$ and $b$ wave scotopic amplitude and Flicker $30 \mathrm{~Hz}$ amplitude) 
Until now, there is no study in Indonesia that reported the normal value of FERG, however compared to Mohamad Rafiudin et $\mathrm{al}^{13}$, our study showed a consistence results with previous study that reported the change in parameter of FFERG limited in OP wave amplitudes, in patients with mild and moderate diabetic retinopathy. In patients with severe NPDR and PDR, prolonged implicit time and significant decreased of Ops amplitude were noted. The decreased of amplitude in $a$ and $b$ wave scotopic and flicker also reported in severe NPDR and PDR. ${ }^{13,14}$ The decrease of OP amplitude can be explained by the pathophysiology of diabetic retinopathy and diabetic macular edema. Histopatologically, in diabetic macular edema, the intracytoplasma Muller cells and outer plexiform layer are swelling. Persistent retinal edema (macular edema) can lead to necrosis of Muller and surrounding neural cells, and eventually forming a cystoid cavity. ${ }^{15-17}$

This research has similarities from previous studies. The primary outcome of our study was the visual acuity improvement, and previous study had reported a transient effect of intravitreal bevazicumab injection in improving visual acuity in DME patients. $^{18-21}$ The improvement of visual acuity in this study was 0.2 LogMar (2 lines ETDRS chart) and the central macular thickness decreased in $19 \%$ at 1 month follow up. Best corrected visual acuity and decreased of central macular thickness at 1 month after injection were statistically significant $(p<0.05)$. Similar results also reported by Haritoglou et al. ${ }^{22}$ There was an improvement in visual acuity from 0.9 LogMar to 0.8 LogMar and $16.9 \%$ decreased in central macular thickness, both at 6 weeks after injection. Arvalo et al. ${ }^{23,24}$ also reported the best visual acuity form 0.9 LogMar to 0.6 LogMar at 2 month after intravitreal injection of bevazicumab. Therefore the results of this study were consistent to the previous studies in order to prove the effectivity of bevazicumab intravitreal injection in DME patients.

Our study showed that in patients with DME, the increased of CMT was related with the worsening of visual acuity although it was not statistically significant. Majority of the patients had a decrease in central macular thickness, but some patients did not experience improvement in visual acuity, even in three patients had a worsening visual acuity. Similar with the study from Lee et $\mathrm{al}^{25}$, which reported a moderate correlation between central macular thickness (OCT scan results) and visual acuity. Browning et $\mathrm{al}^{26}$, concluded that although it had a moderate correlation, the variation of visual acuity to central macular thickness was quite large. Thus, it can be concluded that OCT assessment alone was not sufficient enough as a judgement factor for visual acuity in $\mathrm{DME}^{26}$ We expect that this matters can be explained through electroretinogram examination. In this study there are some factors on the OCT examination were considered in determining visual function. Not only retinal thickness but disorganization of inner retinal layer (DRIL), integrity of photoreceptor IS/OS junction and the regularity of retinal pigment epithelium can also influence the visual function. Some studies mention that diabetic subjects with DRIL have reduced visual acuity and contrast sensitivity more compared to those without DRIL. 
Previous study suggested that to assess the retinal function in DME, MfERG could be the alternative option. The implicit time parameter was considered to be more significant than the amplitude. ${ }^{27}$ The opposite opinion was stated by Greenstein et $\mathrm{al}^{11,28}$, who reported that in patients with clinically significant macula edema/CSME there was a decrease in amplitude and shorter implicit time. Harrison et $\mathrm{al}^{29}$, also stated that both MfERG parameters (implicit time and amplitude) had role in identifying retinal abnormalities in diabetic retinopathy. This was also supported by the International Society for Clinical Electrophysiology and Vision (ISCEV), which stated that MfERG showed a greater amplitude in the fovea due to the number of photoreceptor cone cells and bipolar cells. $^{30}$ Thus, the parameter of MfERG can be used to assess the outer layer of retina and monitor the damage to photoreceptor cells. ${ }^{31}$

In this study the function of macular was measured using the MfERG parameters, including P1, N1, N2 and implicit time of P1, N1, $\mathrm{N} 2$ which were located in the first central macular area (2 degree). MfERG represents the local electrophysiology respond in retinal areas. Firstly, the MfERG would start with negative component (N1) then continued with positive component (P1). These components were respectively in line with $a$ and $b$ wave in FfERG. Our study found that there was a statistically significant decrease in P1 amplitude $(p<0.05)$ at one week post injection, followed with the increase in P1 amplitude but it was not statistically significant $(p>0.05)$ at one month post injection. The result was different with the previous study, which reported that P1 amplitude increased in NPDR patients with diabetic macular edema at one week and two months after intravitreal bevazicumab injection. The reduction of P1 amplitude in our study could be related with the majority of study subjects suffering from proliferative diabetic retinopathy in which there was a progressive damage to Muller cells, bipolar cells and surrounding neural cells. The damage in muller cells and bipolar cells can be illustrated by the decreased in oscillatory potential (OP) wave in FFERG obtained from the baseline data of this study.

Apart from the decreased of P1 amplitude at one week post, there was an improvement in $\mathrm{P} 1$ amplitude at one month post injection which when calculated from one week was statistically significant, but when calculated from pre injection, it was not statistically significant $(p>0,05)$. The improvement of amplitude at one month post injection was consistent with the previous study of Shetty et $\mathrm{al}^{32}$, reported that the increased P1 amplitude at two-month post injection of intravitreal bevazicumab. In addition, Kumar et $\mathrm{al}^{33}$, also stated that the increase in P1 amplitude at one and threemonth after two times injection of intravitreal bevazicumab in DME patients. The difference between our results and the two previous studies could be due to the difference in inclusion criteria, the amount of injection, grading area of MfERG and follow up time, therefore led to different pathophysiology.

The results of this study reported an improvement in implicit time of P1 found in both measurements, although it was not statistically significant. Previous researchers reported that DM patients will show a delay in implicit time due to the local damage of the process in blood glucose mechanism, resulting in delayed neuron transmission. This results were consistent with a study from Greenstein et $\mathrm{al}^{11}$, who reported a prolonged implicit time significantly in diabetic retinopathy patients with CSME versus the healthy group. Abdollah et $\mathrm{al}^{34}$, and Yamamoto et $\mathrm{al}^{35}$, reported the implicit time of P1 prolonged in patients with diabetic macular edema, which indicated a functional deterioration of the outer retinal layers and photoreceptors. The improvement of 
implicit time in P1 wave in this study did not show a statistically significant results, this could be associated with the persistent macular edema and local abnormal (hard exudate) lesions in central macula that were found in several patients. Nonetheless, our results were in line with previous studies which stated that even though there was an implicit time improvement close to normal values, a decrease in visual acuity and amplitude still occurred. ${ }^{36}$ Thus it can be reported that the decrease in visual acuity was not associated with the changes in implicit time. ${ }^{19,37}$

This study showed no significant correlation ( $p>0.05$ ) among the improvement in visual acuity, reduction of CMT, improvement in amplitude and shortening in implicit time of $\mathrm{P} 1$ wave. This was indicated by the presence of individual values that deviate from the expected results, in 2 patients who experienced deterioration in visual acuity even though the CMT was close to normal < 300 but MfERG parameter had worsened. Meanwhile, 2 patients had improvement in visual acuity along with MfERG parameters although the CMT still high. Dale et $\mathrm{al}^{38}$, argued that there was a fundamental difference between OCT and MfERG, in which MfERG tends to mistakenly detect small local abnormalities that can be detected with OCT. Therefore, the functional damage can be detected by MfERG before structural changes occur, thus it can be concluded that OCT and MfERG assessments were complementary as predictors of visual acuity in DME patients. ${ }^{38}$ Clinically, OCT and MfERG examinations were very useful tools to identify the location and the degree of retinal damage.

\section{CONCLUSION}

There was no correlation among the decrease of central macular thickness, improvement of P1 wave amplitude, and shorter implicit time in $\mathrm{P} 1$ wave with improvement of visual acuity in patients with DME post anti-VEGF intra vitreal injection. Anti-VEGF intravitreal injection had a significant depletion in $\mathrm{P}$ wave amplitude after 1 week of injection, followed with the improvement of $\mathrm{P}$ wave amplitude, however it was not statistically significant after one month of injection. Anti-VEGF intravitreal injection improved the implicit time of $\mathrm{P}$ wave at one month after injection, but it was not statistically significant. AntiVEGF intravitreal injection was statistically significant to decrease the central macular thickness onemonth after injection. Anti-VEGF intravitreal injection statistically significant to improve the visual acuity one-month after injection.

The limitation of this study are the variability of the sample size was quite large, therefore the distribution of pre injection subjects was abnormal. Inclusion criteria should be more limited by ranging the BCVA patients in $1 / 60 \leq x \leq 6 / 60$ and $6 / 60<$ to $\leq 6 / 9$. Future studies with a large number of subjects are needed to obtain a better research result. The SD value on this study was 309 with a difference in amplitude 129.95, therefore the total sample needed for the future study should be around 44 patients.

\section{ACKNOWLEDGMENT}

The authors thank all patients who participated in this study, Rianti Wulandari Pratiwi and Dhiny Lidinillah as research assistant.

\section{REFERENCES}

1. Pascolini D, Mariotti SP. Global estimates of visual impairment: 2010. British Journal of Ophthalmology. 2012;96(5):614-8.

2. Klein BEK. Overview of epidemiologic studies of diabetic retinopathy. Ophthalmic Epidemiology. 2007;14(4):179-83.

3. Klein $R$, Klein BEK, Moss SE. The Wisconsin epidemiologic study of diabetic retinopathy. IV. Diabetic macular edema. Ophthalmology. 1984;91(12):1464-74.

4. Group ETDRSR. Early photocoagulation for diabetic retinopathy ETDRS report. Ophthalmology. 1991;98(5):766-85. 
5. Fong D, Strauber S, Aiella L, Beck R, Callanan D, Danis $\mathrm{R}$, et al. Comparison of the modified Early Treatment Diabetic retinopathy Study and mild macular grid laser photocoagulation strategies for diabetic macular edema. Arch Ophthalmol. 2007;125:469-80.

6. Michaelides $M$, Kaines $A$, Hamilton RD, Fraser-Bell $S$, Rajendram $R$, Quhill $F$, et al. A Prospective Rabdomized Trial of INtravitreal Bevacizumab or Laser Therapy in the Management of Diabetic Macular Edema (BOLT Study). Ophthalmology. 2010;117:107886.

7. Regnier S, Malcolm W, Allen F, Wright J, Bezylax V. Efficacy of Anti-VEGF and Laser Photocoagulation in the Treatment of Visual Impairment due to Diabetic Macular Edema: A Systematic Review and Network Meta-Analysis. PLOS ONE. 2014;9(7):1-10.

8. Lai TYY, Chan W-M, Lai RYK, Ngai JWS, Li H, Lam DSC. The Clinical Applications of Multifoca Electroretinography: A Systematic Review. Survey of Ophthalmology. 2007;52(1):61-96.

9. Jr MAB, Adams AJ, Han Y, Schneck ME, Ng J, BronsonCastain $\mathrm{K}$, et al. A multifocal electroretinogram model predicting the development of diabetic retinopathy. Retinal and eye Research. 2006;25:425-48.

10. Brodie SE, Naidu EM, Goncalves J. Combined Amplitude and Phase Criteria for Evaluation of Macular Electroretinograms. Ophthalmology. 1992;99:522-30.

11. Greenstein V, Holopigian K, Seiple W, Carr R, Hood D. Atypical multifocal ERG responses in patients with diseases affecting photoreceptors. Vision Research. 2004:44:2867-74.

12. Farahvash MS. Multifocal Electroretinogram in Clinically Significant Diabetic Macular Edema. Archives of Iranian Medicine. 2006;9(3):261-5.

13. Tzekov R, Arden GB. Electroretinogram in Diabetic Retinopathy. Survey of Ophthalmology. 1999;44(1):53-60.

14. Maturi RK, Bleau LA, Wilson DL. Electrophysiologic findings after intravitreal bevaciumab (avastin) treatment. Retina. 2006;26:270-4. 21.

15. Pezek C LJ. Diabetic Macular Edema: review and update Ophthalmol CLin North Am. 2002;15:555-63.

16. Das A, McGuire P, Rangasamy S. Diabetic Macular Edema: Pathophysiology and Novel Therapeutic Targets. Ophthalmology. 2015;1:1-20.

17. Brownlee $M$. The pathobiology of diabetic complications: a unifying mechanism. Diabetes. 2005;54:1615-25.

18. Higashida $R$, Imamura $Y$, Ishikawa $A$, Tsutsumi $Y$, Ichikawa $Y$, Wakakuri $T$, et al. Intravitreal injection of Bevacizumab for diabetic macula edema: morphological and functional outcomes at 1 day after injection. Investigative Ophthalmology \& Visual Science. 2014;55:1764.

19. Sivaprasad S, Crosby-Nwaobi R, Esposti S, Rajendram TP, Michaelides M, Hykin P. Structural and Functional Measures of Efficacy in Response to Bevacizumab Monotherapy in Diabetic Maclar Oedema: Exploratory Analyses of the BOLT Study (report 4). PLOS ONE. 2013;8(8):1-7.

20. Nourinia R, Azarmina $M$, Soheilian M. Intravitreal Bevacizumab for Treatment if Diabetic Macular Oedema. European Ophthalmic review. 2013;7(1):4551.

21. Scott I, Edwards A, Beck R, Bressler N, Chan C, Elman $M$, et al. A phase II randomized clinical trial of intravitreal bevacizumab for diabetic macular edema.Ophthalmology.2007;114:1860-7.

22. Haritoglou C, Kook D, Neubauer A, Wolf A, Priglinger $S$, Strauss $R$, et al. Intravitreal bevacizumab (Avastin) therapy for persistent diffuse diabetic macular edema. Retina. 2006;26:999-1005.

23. Arevalo J, J JF-G, Quiroz-Mercado H, JG Sanchez JG, Wu L, Maia $M$, et al. Primary intravitreal bevacizumab (avastin) for diabetic macular edema: results from the Pan-American Collaborative Retina Study Group at 6month follow-up.. Ophthalmology. 2007;114:743-50.

24. Arevalo J, Lasave A, Wu L, Diaz-Llopis M, GallegoPinazo R, Alezzandrini A. Pan-American Collaborative Retina Study Group (PACORES).Intravitreal Bevacizumab plus grid laser photocoagulation or intravitreal Bevacizumab or grid laserphotocoagulation for diffuse diabetic macular edema: results of the Pan-american Collaborative Retina Study Group at 24 months. Retina. 2013;33(2):403-13.

25. Lee K, Chung H, Park Y, Sohn J. Efficacy of Intravitreal Anti-vascular Endhotelial Growth Factor or Steroid Injection in Diabetic Macular Edema According to Fluid Turbidity in Optical Coherence Tomography. Korean Journal of Ophthalmology. 2014;28(4):298305.

26. DJ DB, Glassman A, Aiello L, Beck R, Brown D, Diabetic Retinopathy Clinical Research Network. Relationship between optical coherence tomography-measured central retinal thickness and visual acuity in diabetic macular edema. Opthalmology. 2007;114:526-36.

27. Fortune B, Schneck $M$, Adams A. Multifocal electroretinogram delays reveal local retinal dysfunction in early diabetic retinopathy.Invest Ophthalmol Vis Sci. 1999;40:2638-51.

28. Greenstein V, Holopigian K, Hood DC, Seiple W, Carr $R$. The nature and extent of retinal dysfunction associated with diabetic macular edema. . Invest Ophthalmol Vis Sci. 2000;41:3643-54. 
29. Harrison W, Jr MB, Schneck M, Wolff B, Jewell N, Barez $S$. Prediction, by retinal location, of the onset of diabetic edema in patients with nonproliferative diabetic retinopathy. Invest Ophthalmol Vis Sci. 2011;52:6825-31.

30. Hood D, Bach M, Brigell M, Keating D, Kondo M, Lyons J. ISCEV standard for clinical multifocal electroretinography (mfERG) (2011 edition). Doc Ophthalmol. 2012;124:1-13.

31. Hood DC. Assessing retinal function with the multifocal technique. Prog Retin Eye Res. 2000;19:607-46.

32. Shetty R, Pai SA, Vincent A, Shetty N, Narayana KM, Sinha $B$, et al. Electrophysiological and strusctural assessment of central retin following intravitreal injection of bevacizumab for treatment of macular edema Doc Ophthalmol. 2008;116:129-35.

33. Kumar A, Sinha S. Intravitreal Bevacizumab(Avastin) treatment of diffuse diabetic macular edema in an indian population. . Indian J ophthalmol. 2007;55(6):451-5.

34. Abdollahi A, Movassa M, Ahmadabadi M, Abdollahi M, A AAB. Multifocal electroretinography assisted comparison of macular photocoagulation versus macular photocoagulation and intravitreal
Bevacizumab injection in diabetic macular edema . Iranian Journal of Ophthalmology 2010;22(3):23-8.

35. Yamamoto S, Yamamoto T, Hayashi M, Takeuchi S. Morphological and functional analyses of diabetic macular edema by optical coherence tomography and multifocal electroretinograms. Graefe's Arch Clin Exp Ophthalmol. 2001;239:96-101.

36. Ozkiris A. Pattern electroretinogram changes after intravitreal bevacizumab injection for diabetic macular edema. Doc Ophthalmol. 2010;120:243-50.

37. Palmowski AM, Sutter EE, Marcus A. Bearse J, Fung W. Mapping of Retinal Function in Diabetic Retinopathy Using the Multifocal Electroretinogram. Investigative Ophthalmology \& Visual Science 1997;38(12):258696.

38. Dale E, Hood D, Greenstein V, Odel J. A comparison of multifocal ERG and frequency domain OCT changes in patients with abnormalities of the retina. Doc Ophthalmol. 2010;120:175-86.

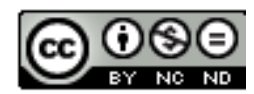

This work licensed under Creative Commons Attribution 